\title{
Overweight or obesity are not risk factors for central venous catheter-related bloodstream infections in patients with hematological malignancies
}

\author{
Enrico Schalk $M D^{1}$ (1) and Marcus Hentrich $\mathrm{MD}^{2}$ (1) \\ ${ }^{1}$ Department of Hematology and Oncology, Otto-von-Guericke University Magdeburg, Medical Center, Magdeburg, Germany and ${ }^{2}$ Department of Hematology and \\ Oncology, Red Cross Hospital Munich, Munich, Germany
}

To the Editor-In 2015, we reported on the risk of central venous catheter (CVC)-related bloodstream infections (CRBSIs) in obese patients with hematological malignancies. ${ }^{1}$ Considering together definite, probable, and possible CRBSIs, ${ }^{2}$ we detected no difference in the CRBSI rate between obese and nonobese patients $(22.1 \%$ vs $23.3 \%){ }^{1}$ However, this analysis was based on only 335 CVC cases from the Magdeburg cohort of the SECRECY registry (German Clinical Trial Register, no. DRKS00006551). ${ }^{3}$ Furthermore, we did not distinguish between obese and overweight patients. Although both obesity and overweight are associated with increased disease-specific morbidity for some diseases, obesity mostly confers a higher risk than overweight. ${ }^{4}$ Therefore, we updated our analysis on the CRBSI risk in both obese and overweight patients from the Magdeburg cohort of the SECRECY registry.

Patients with a body mass indexes (BMIs) of $18.5-24.9 \mathrm{~kg} / \mathrm{m}^{2}$ were considered normal weight, those with $25.0-29.9 \mathrm{~kg} / \mathrm{m}^{2}$ were considered overweight, and patients with a BMI $\geq 30 \mathrm{~kg} / \mathrm{m}^{2}$ were considered obese. ${ }^{5}$ For a diagnosis of definite or probable CRBSI we used the 2012 definitions of the Infectious Diseases Working Party (AGIHO) of the German Society of Hematology and Medical Oncology (DGHO). ${ }^{2}$ From March 2013 to September 2021, 1,046 nontunneled jugular or subclavian vein CVCs were inserted in patients with a BMI of $>18.5 \mathrm{~kg} / \mathrm{m}^{2}$. Patient and CVC characteristics as well as CRBSI features are shown in Table 1. A median of $1 \mathrm{CVC}$ was inserted per patient (mean, 1.9; range, 1-7). In 699 cases (66.8\%), CVCs were inserted in patients with BMI $\geq 25 \mathrm{~kg} / \mathrm{m}^{2}: 405$ (38.7\%) were overweight and 294 (28.1\%) were obese. The median BMI of both overweight and obese patients was $26 \mathrm{~kg} / \mathrm{m}^{2}$ (range, 19-56). With respect to the entire group of patients, $21.7 \%$ were neutropenic at time of CVC insertion and $87.6 \%$ were neutropenic at at CRBSI onset, with no significant differences between groups. Most CVCs were inserted in the internal jugular vein and had chlorhexidine-coated CVC dressings.

The median number of CVC days was lower in obese compared to normal weight or overweight patients (14 vs 17 days). However, the median time to CRBSI onset was similar between the groups (11 and 12 days, respectively). The CRBSI rate was significantly higher in the overweight as compared to the normal weight group

Author for correspondence: Enrico Schalk, E-mail: enrico.schalk@med.ovgu.de Cite this article: Schalk E and Hentrich M. (2022). Overweight or obesity are not risk factors for central venous catheter-related bloodstream infections in patients with hematological malignancies. Infection Control \& Hospital Epidemiology, 43: 1953-1955, https://doi.org/10.1017/ice.2021.491
(12.1\% vs $7.5 \%$; hazard ratio [HR], 1.66; $95 \%$ confidence interval [CI], 1.03-2.68; $P=.04$ ), whereas it was not significantly different between obese and normal weight patients (10.2\% vs $7.5 \%$; HR, 1.26; 95\% CI, 0.97-1.64; $P=.09$ ).

Furthermore, the incidence of CRBSI was higher in overweight than the normal weight patients (7.4 per 1,000 CVC days vs 4.5 per 1,000 CVC days; $P=.04$ ) but not in obese compared to normal weight patients (7.0 per 1,000 CVC days vs 4.5 per 1,000 CVC days; $P=.10)$.

These findings are somewhat surprising. Notably, the proportion of men in the overweight group was higher than in the obese group $(68.1 \%$ vs $55.1 ; P<.001)$. Male sex was previously identified as an independent risk factor for CRBSI in patients with hematological malignancies, ${ }^{6}$ and this may have contributed to the higher CRBSI rate and incidence in the overweight group. However, in another sex-matched analysis of the whole cohort, we detected no significant difference in the CRBIS rate between the overweight and the normal weight group [22 (8.5\%) of 258 vs 23 (7.7\%) of 298 ; $P=.73$ ] nor between the obese and the normal weight group [26 (9.8\%) of 264 vs $23(7.7 \%)$ of $298 ; P=.38$ ]. In addition, we detected no difference in the CRBSI incidence between the overweight and the normal weight group (5.1 per 1,000 CVC days vs 4.7 per $1,000 \mathrm{CVC}$ days; $P=.76$ ) nor between the obese and the normal weight group (6.7 per 1,000 CVC days vs 4.7 per $1,000 \mathrm{CVC}$ days; $P=.20)$.

In summary, our results show that neither obesity nor overweight are risk factors for CRBSI in patients with hematological malignancies. However, we cannot rule out that both potential risk factors were merely masked by neutropenia, a well-established risk factor for CRBSI in hematological patients, ${ }^{2,7}$ which was present in $\sim 90 \%$ at CRBSI onset in our cohort. Apart from this, obesity has been shown to be an independent risk factor for CRBSIs in the ICU setting, ${ }^{8,9}$ possibly because of inadequate CVC dressings or CVC dressing disruption..$^{9,10}$

Acknowledgments. The authors would like to thank all colleagues of the Magdeburg cohort of the SECRECY registry for their great support and documentation.

Financial support. No financial support was provided relevant to this article.

Conflicts of interest. E.S. reports personal fees from Gilead Sciences, Stemline Therapeutics Switzerland, Carl-Thiem-Klinikum Cottbus, Ärztekammer Sachsen-Anhalt, LIAM, and Forschung und Lehre in der KGHI outside the submitted work. M.H. reports personal fees from Amgen, BMS, Eusa Pharma, Janssen, Jazz Pharma, Sanofi, Takeda, and Celgene during the study. 
Table 1. Patients/CVCs Characteristics and CRBSI Features

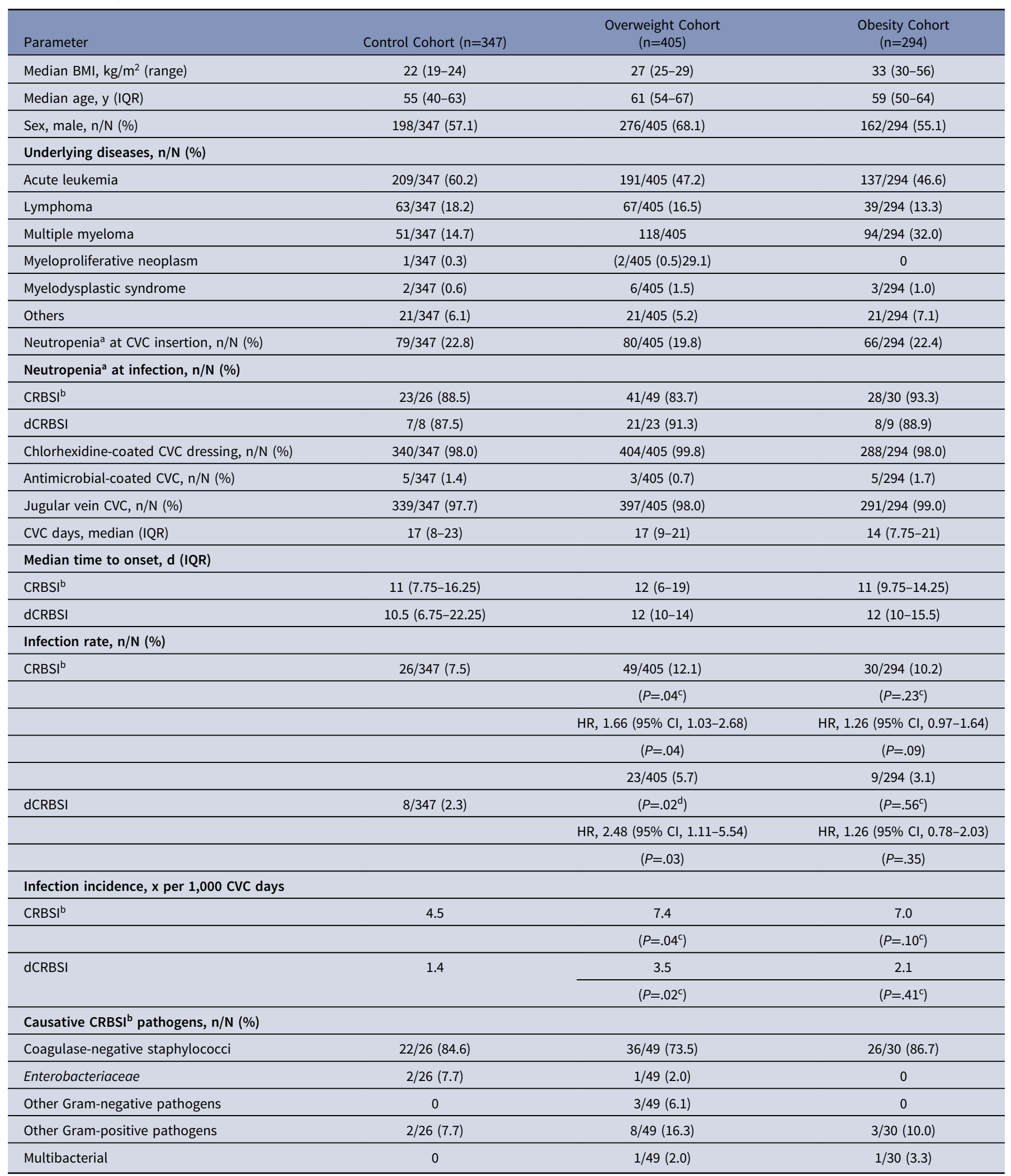

Note. CVC, central venous catheter; CRBSI, central venous catheter-related bloodstream infection; dCRBSI, definite CRBSI'; BMI, body mass index; IQR, interquartile range; HR, hazard ratio; CI, confidence interval. All $P$ values are 2 -sided.

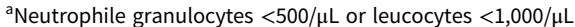

${ }^{b}$ Combined definite or probable CRBSI. ${ }^{2}$

${ }^{\mathrm{c}}$ Mid- $P$ exact test. 


\section{References}

1. Schalk E, Färber J, Fischer T, Heidel F. Central venous catheter-related bloodstream infections in obese hematologic patients. Infect Control Hosp Epidemiol 2015;36:995-996.

2. Hentrich M, Schalk E, Schmidt-Hieber M, et al. Central venous catheterrelated infections in hematology and oncology: 2012 updated guidelines on diagnosis, management and prevention by the Infectious Diseases Working Party of the German Society of Hematology and Medical Oncology. Ann Oncol 2014;25:936-947.

3. Schalk E, Hanus L, Färber J, Fischer T, Heidel FH. Prediction of central venous catheter-related bloodstream infections (CRBSIs) in patients with hematologic malignancies using a modified Infection Probability Score (mIPS). Ann Hematol 2015;94:1451-1456.

4. Lenz M, Richter T, Mühlhauser I. The morbidity and mortality associated with overweight and obesity in adulthood: a systematic review. Dtsch Arztebl Int 2009;106:641-648.

5. Anonymous. Clinical guidelines on the identification, evaluation, and treatment of overweight and obesity in adults - the evidence report. National Institutes of Health. Obes Res 1998;6 suppl 2:51S-209S.
6. Schalk E, Toelle D, Schulz S, et al. Identifying haematological cancer patients with high risk for central venous catheter (CVC)-related bloodstream infections at the time point of CVC insertion. 29th European Congress of Clinical Microbiology and Infectious Diseases, April 13-16, 2019, Amsterdam, The Netherlands, abstract P2556. https://www.escmid.org/escmid_publications/ escmid_elibrary. Published 2019. Accessed October 9, 2021.

7. Tölle D, Hentrich M, Pelzer BW, et al. Impact of neutropenia on central venous catheter-related bloodstream infections in patients with hematological malignancies at the time of central venous catheter insertion: a matchedpair analysis. Infect Control Hosp Epidemiol 2019;40:1204-1206.

8. Dossett LA, Dageforde LA, Swenson BR, et al. Obesity and site-specific nosocomial infection risk in the intensive care unit. Surg Infect (Larchmt) 2009;10:137-142.

9. Buetti N, Souweine B, Mermel L, et al. Obesity and risk of catheter-related infections in the ICU. A post hoc analysis of four large randomized controlled trials. Intensive Care Med 2021;47:435-443.

10. Trick WE, Miranda J, Evans AT, Charles-Damte M, Reilly BM, Clarke P. Prospective cohort study of central venous catheters among internal medicine ward patients. Am J Infect Control 2006;34:636-641.

\title{
Trends in antimicrobial susceptibility patterns in healthcare-associated methicillin-resistant Staphylococcus aureus from bloodstream infections: A joinpoint regression analysis
}

\author{
Diana Mayumi Nii MS, Maria Aparecida Vitagliano Martins MD and Carlos Magno Castelo Branco Fortaleza MD, PhD (1) \\ Department of Infectious Diseases, Botucatu School of Medicine, São Paulo State University (UNESP), Botucatu, São Paulo State, Brazil
}

To the Editor-Staphylococcus aureus ranks third among pathogens causing healthcare-associated bloodstream infections in Brazil, and $>60 \%$ of reported isolates are methicillin resistant (ie, MRSA). ${ }^{1}$ The rise of healthcare-associated MRSA in Brazil occurred in the 1990s, mostly due to the extensive spread of the Brazilian epidemic clone (BEC). ${ }^{2}$ BEC harbored the staphylococcal chromosome cassette (SCC) mec type III and were typically resistant to several antimicrobials, such as trimethoprim/ sulfametoxazole (TMP/SMX), quinolones, and clindamycin. For a long time (before the national registration of linezolid and daptomycin), glycopeptides remained as the sole therapeutic option for healthcare-associated MRSA (HA-MRSA) in Brazil. ${ }^{3}$

Recent studies report that BEC has been substituted for clones harboring SCCmec type II, with remarkable increasing susceptibility to TMP/SMX and modest increases in susceptibility to ciprofloxacin and clindamycin. ${ }^{4,5}$ Sporadic findings have indicated that TMX/SMX-susceptible, SCCmec type IV-harboring MRSA clones, which probably originated in the community, have spread within Brazilian hospitals. ${ }^{6,7}$ Susceptibility to TMP/ SMX, ciprofloxacin, and clindamycin has been proposed as a proxy marker of the so-called community-associated MRSA (CA-MRSA) invading hospitals. ${ }^{8}$

Author for correspondence: Carlos Magno Castelo Branco Fortaleza, E-mail: carlos. fortaleza@unesp.br

Cite this article: Nii DM, Martins MAV, and Fortaleza CMCB. (2022). Trends in antimicrobial susceptibility patterns in healthcare-associated methicillin-resistant Staphylococcus aureus from bloodstream infections: A joinpoint regression analysis. Infection Control \& Hospital Epidemiology, 43: 1955-1957, https://doi.org/10.1017/ ice. 2021.448
Time series analysis, especially joinpoint regression techniques, which detect changes in time trends, have been rather infrequently applied to analyze long-term trends in antimicrobial resistance within healthcare settings. With that in mind, we conducted a time series analysis of HA-MRSA bloodstream infections (BSIs) in a teaching hospital from inner Brazil. The Botucatu Medical School teaching hospital has 500 beds and is a tertiary-care referral facility for an area with 500,000 inhabitants. Briefly, we analyzed monthly proportions of resistance to TMP/SMX, clindamycin, and ciprofloxacin among CA-MRSA BSIs from 2005 through 2019. During that 15 years, 2,291 nonduplicate episodes of CA-MRSA BSI were detected. We used Joinpoint version 4.9 software (National Cancer Institute, Calverton, MD) to identify changes in the time trends of those resistance patterns. We used a linear approach, and a minimum interval of 6 months between joinpoints was selected.

Our results are summarized in Figure 1. The overall resistance rates were as follows: TMP/SMX, 26.6\%; clindamycin, 77.6\%; and ciprofloxacin, $73.5 \%$. We found 3 joinpoints for TMP/SMX resistance; the most relevant was followed by an abrupt decrease from $80.2 \%$ to $41.0 \%$ beginning in August 2007. The trend changed to a slower decrease until June 2016 (to $37.5 \%$ ), with a small increase thereafter. Both clindamycin (1 joinpoint in July 2014) and ciprofloxacin (2 joinpoints in February and September 2011, respectively) presented initial decreases followed by slow increase in resistance. Notably, joinpoints in trends were not simultaneous for different antimicrobials.

This picture is more compatible with the substitution of SCCmec type III-harboring BEC for SCCmec II-harboring clones, which has been reported to maintain high levels of resistance to 